\title{
Measurement-Based Analysis of the Performance of several Wireless Technologies
}

\author{
R. Cosma ${ }^{\mathrm{d}}$, Albert Cabellos-Aparicio ${ }^{\mathrm{a}}$, J. Domenech-Benlloch ${ }^{\mathrm{b}}$, J. Gimenez-Guzman $^{\mathrm{b}}$, \\ J. Martinez-Bauset ${ }^{b}$, M. Cristian ${ }^{d}$, A. Fuentetaja ${ }^{c}$, A. López $^{\mathrm{a}}$, J. Domingo-Pascual ${ }^{\mathrm{a}}, \mathrm{J}_{\text {. Quemada }}{ }^{\mathrm{c}}$ \\ a. Universitat Politècnica \\ de Catalunya \\ Dept. of Computer \\ Architecture \\ c/ Jordi Girona 1-3, \\ 08034 Barcelona, Spain \\ c. Universidad Politécnica \\ de Madrid \\ Dept. de Ingeniería de \\ Sistemas Telemáticos \\ Ciudad Universitaria $\mathrm{s} / \mathrm{n}$. \\ 28040 Madrid, Spain \\ d. Technical University of \\ Cluj-Napoca \\ Dept. of Communications \\ 26-28 George Baritiu St., \\ 400027 Cluj-Napoca, \\ Romania
}

\begin{abstract}
Wireless technologies have rapidly evolved and are becoming ubiquitous. An increasing number of users attach to the Internet using these technologies; hence the performance of these wireless access links is a key point when considering the performance of the whole Internet. In this paper we present a measurement-based analysis of the performance of an IEEE 802.16 (WiMAX) client and an UMTS client. The measurements were carried out in a controlled laboratory. The wireless access links were loaded with traffic from a multi-point videoconferencing application and we measured three layer-3 metrics (One-Way-Delay, IP-Delay-Variation and Packet Loss Ratio). Additionally we estimate the performance of a WiFi and Ethernet client as a reference. Our results show that Ethernet and WiFi have comparable performances. Both the WiMAX and the UMTS links exhibited an asymmetric behavior, with the uplink showing an inferior performance. We also assessed the causes of the discretization which appears in the jitter distributions of these links.
\end{abstract}

Index Terms-Measurement, Testbed, WiMAX, UMTS, Empirical analysis

\section{INTRODUCTION}

$\mathrm{W}$ IRELESS technologies have rapidly evolved in recent years. Nowadays, IEEE 802.11 (WiFi) [1] is one of the most used wireless technologies and it provides up to $54 \mathrm{Mbps}$ of bandwidth in an easy and affordable way. This technology has been deployed worldwide, from campus to commercial networks and it is becoming ubiquitous.

Recently new wireless technologies have been researched, and deployed. Currently UMTS [2] and IEEE 802.16 [3] (WiMAX) are among the new wireless technologies that stand out. These technologies provide more bandwidth, greater range and are intended to be used as last-mile access links. Additionally these new technologies incorporate mobility. With mobility, a user can connect to the Internet, and move within a limited geographical area without breaking its IP communications.

This work is a collaborative research partially funded by the MEC (Spanish Ministry of Education and Science) under contract TSI 2005-07520-C03. Additionally we would like to thank funding by IST under contract IST2006-NoE-0384239 (IST-CONTENT) and the CIRIT (Catalan Research Council) under contract 2005-SGR-0048.
Currently an increasing number of users are attached to the Internet using one of these technologies. The end-to-end paths used by the user's connections include one, or even two of these wireless access links. That's why we believe that the performance of these technologies is a key point when considering the performance of the whole Internet.

The research community has focused on measuring the performance of these technologies. Initially A. Mishra presented in [4] a measurement-based analysis of the performance of different IEEE 802.11 network interfaces cards. The authors specifically focused on the handover process of WiFi-based networks. Later many other authors provided different analysis, at different levels, of the performance of WiFi $[5,6]$ (and the references therein).

Regarding UMTS, some authors have also measured the performance of these links. An in-depth packet delay analysis and a model for the UMTS packet delay were proposed in [7] and an extensive measurement study on several commercial networks was conducted in [8].

Finally regarding WiMAX, the research community has started to provide empirical measurement-based analysis of its performance. In [9] the authors measured propagation loss at a specific frequency. A model for link throughput based on received signal strength was proposed in [10] and in [11] an experimental WiMAX link was subjected to different load conditions and tested from a transport layer point of view. Mobility performance with the link subjected to various physical phenomena (multi-path, Doppler shift, etc.) was carried out in [12].

In this paper we present a measurement-based analysis of the above-mentioned wireless access technologies. We determine the performance, at the IP layer, of an IEEE 802.11 (WiFi), a commercial UMTS (using HSPA) and an experimental IEEE 802.16 (WiMAX) access link. Additionally we measure the performance of an Ethernet client as a reference. To the best of the author's knowledge this is the first work that summarizes comparatively the performances of all these technologies. We base our evaluation on the following metrics: One-Way-Delay (OWD), Packet Loss Ratio (PLR) and Inter-Packet Delay Variation

Proceedings of the 2008 16th IEEE Workshop on Local and Metropolitan Area Networks 
(IPDV). These metrics have been defined in RFC 2679, RFC 3393 and RFC 2680 and are considered as standard metrics when measuring the performance of Internet data delivery services.

Our tests were carried out in a distributed laboratory across three Spanish universities (UPC, UPV and UPM) interconnected by the RedIris academic network. Instead of using synthetic traffic to load the different access links we used a video-conferencing application: Isabel [14]. Isabel is intended for large multipoint configurations and it uses an overlay star topology: all the flows are forwarded through a central server (flow-server). During a experiment we run Isabel for 900 s with all the clients connected simultaneously. We capture all the packets sent/received at the clients and at the flow-server. With these captures, we are able to compute the above-mentioned metrics using the passive measurement methodology. We also give an empiric description of the QoE based solely on user experience.

Our results show that the performance of the WiFi client is comparable to that of Ethernet. We have also found that the WiMAX client experiences an asymmetric behavior and high packet loss. The UMTS client outperforms the WiMAX client in terms of delay but its low throughput imposes certain limitations. The nature of jitter on both links is discrete due to the MAC Layer retransmission schemes employed.

This paper is structured as follows: Section 2 provides details about the utilized test-bed, the passive measurement methodology and an overview of the captured traffic used in our analysis. Section 3 discusses the measurement results and their implications for the wireless link characterization. Finally, section 4 summarizes the main conclusions.

\section{Methodology}

This subsection details the test-bed deployed to collect the measurements, the methodology used to compute the performance metrics and provides an overview of the captured traffic used in the following analysis.

\section{A. Test-Bed}

Figure 1 presents our distributed test-bed among three universities (UPC, UPM and UPV). All three universities are connected through RedIris, the Spanish academic research network (NLANR).

The test-bed includes five terminals. Three of them are equipped with an IEEE 802.11, an IEEE 802.16 and an UMTS interface. Another one is equipped with an Ethernet (as reference). The last one is the Isabel flow-server. All the machines are Linux-based, at least Pentium III with 1GB of RAM.

Isabel uses an overlay network to transmit live audio and video streams. All the streams are routed through the flowserver, creating an overlay star-topology. Each client transmits both audio and video, which is received by the rest of the clients. The microphone and the camera of each client transmit a movie, to setup a realistic environment.

Additionally each terminal captures all the incoming and outgoing traffic using a PCAP-based [15] application. Later

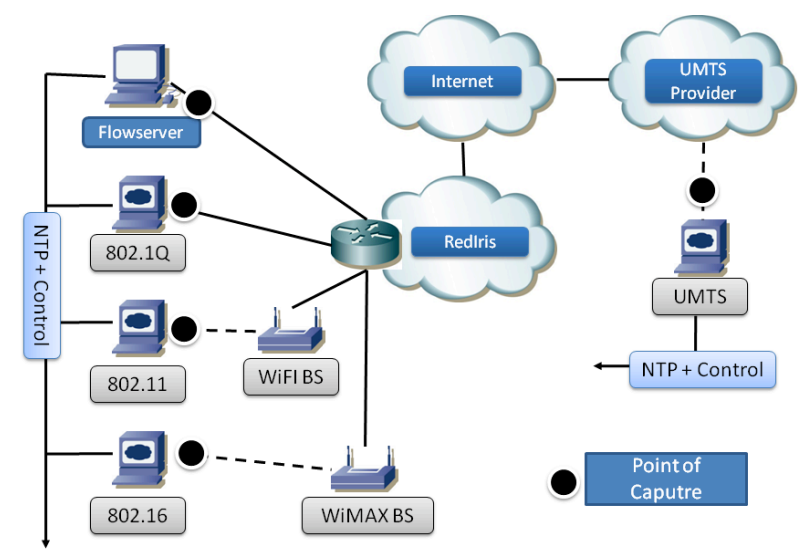

Fig. 1. Test-bed Configuration

this traffic is analyzed offline to compute the metrics.

Regarding synchronization each client is configured to use three NTP (Network Time Protocol) sources [16]. At least each client is connected to a Stratum 1 server which is in turn connected to a GPS source. The other two sources are on the outside network. All the NTP traffic is routed through a parallel network (with the local NTP servers). It is possible to access those remote NTP servers through the control network that can use external time sources. The NTP statistics show that, with this setup, we obtain a measurement accuracy of $1 \mathrm{~ms}$. Since the delays of the different measured links are higher this accuracy suffices.

\section{B. Passive Measurement Methodology}

The main focus of the tests was the estimation of the following metrics: One-way Delay (OWD), IP Delay Variation (Jitter), and Packet Loss Ratio (PLR). With this range of parameters we can determine the ability, reliability and the robustness of each wireless technology:

OWD represents the time that a packet takes to travel through the network from source to destination. For a realtime application, it is important that this parameter stays below a threshold value (RFC 2679).

IPDV refers to the variation of a packet's one-way delay in respect to the one-way delay of the previous packet (we assume here that both packets belong to the same flow). Erratic variation in delay makes it difficult (or impossible) to support many real-time applications (RFC 3393).

PLR is a percentage ratio of the number of data packets lost to the total number of packets transmitted by the user into the network (RFC 2680).

As pointed out earlier, the probe traffic was generated using a videoconferencing application. This approach allows us to estimate the performance of the network technologies using both the Constant Bit Rate (CBR) nature of the audio stream and the Variable Bit Rate (VBR) nature of the video stream.

Isabel uses the Real-time Transport Protocol (RTP) over the User Datagram Protocol (UDP) to send its data. As shown in [10], UDP is the most appropriate protocol for determining the 
uplink/downlink performance of an access technology, since the lack of acknowledgments eliminates the interdependency between the bitrates in the two directions.

The port numbers assigned for the transmission of audio and video flows are known, and the SSRC (Synchronization Source) field of the RTP header has an unique value for each client. Based on this information we developed a custom tool that can load and match packets from the source and destination capture files, even if the packets passed through a point where Network Address Translation was used. The tool uses PCAP's timestamp from each matched packet pair to compute the aforementioned metrics.

\section{Captured Data Overview}

The traffic was captured for the subsequent offline analysis during two test sessions, each one with a duration of approximately 900 seconds.

We used two profiles for the videoconferencing application with different quality settings. The high quality profile was switched on for 300 seconds during each test, and the rest of the test was conducted with the lower quality profile.

During a test session Isabel generated $\sim 21000$ and $\sim 32000$ packets corresponding to average bitrates of $111.212 \mathrm{kbps}$ and $69.684 \mathrm{kbps}$ for the video and audio flows, respectively.

\section{RESULTS}

\section{A. Ethernet}

Table I shows the minimum, maximum, mean, median and standard deviation of the one-way delay and the jitter experienced between the Ethernet client and the flow-server. These parameters are evaluated using the video and audio streams in both the uplink and the downlink directions. The minimum and maximum values are taken as the 2.5 and 97.5 percentiles of the distributions.

TABLE I

ETHERNET CLIENT METRICS STATISTICS

\begin{tabular}{|c|c|c|c|c|c|c|}
\hline \multicolumn{2}{|c|}{ Metric } & Max & Mean & Median & Min & Dev \\
\hline \multirow{2}{*}{$\begin{array}{c}\text { Video } \\
\text { Up }\end{array}$} & OWD & 11.5483 & 9.7942 & 9.49407 & 8.9369 & 1.2901 \\
\cline { 2 - 7 } & IPDV & 0.3541 & 0 & 0.02193 & -1.0117 & 1.0018 \\
\hline Audio & OWD & 9.4810 & 9.0133 & 8.89993 & 8.6751 & 0.6347 \\
\cline { 2 - 7 } Up & IPDV & 0.3221 & 0 & 0.01597 & -0.6061 & 0.6613 \\
\hline $\begin{array}{c}\text { Video } \\
\text { Down }\end{array}$ & OWD & 8.7628 & 8.4993 & 8.56996 & 7.8580 & 0.3236 \\
\cline { 2 - 7 } & IPDV & 0.6180 & 0 & $-405 \mu \mathrm{s}$ & -0.7380 & 0.3637 \\
\hline $\begin{array}{c}\text { Audio } \\
\text { Down }\end{array}$ & OWD & 8.2281 & 8.0075 & 7.97701 & 7.8502 & 0.1580 \\
\cline { 2 - 7 } & IPDV & 0.1991 & 0 & $-95 \mu \mathrm{s}$ & -0.3399 & 0.1745 \\
\hline
\end{tabular}

All values are in milliseconds, unless specified otherwise.

In the uplink direction this client performed well, with a low median (50 percentile) value of the OWD $(9.49 \mathrm{~ms})$. The IPDV distribution is zero-centered with a median value of under $0.03 \mathrm{~ms}$. The PLR was $0.088 \%$ for the video stream and $0.006 \%$ for the audio stream.

In the downlink direction the client had to receive audio and video streams from all other three clients. Nevertheless its performance remained high, scoring a $8.499 \mathrm{~ms}$ median value for the OWD and a well-shaped IPDV distribution. However, we recorded a higher PLR of $0.507 \%$ for the video streams and $0.006 \%$ for the audio streams.
When we analyzed the OWD and IPDV in respect to the packet length, we saw a slight increase of the IPDV $(0.15 \mathrm{~ms})$ as the packet size increased from 100 to 900 bytes and a relatively flat OWD characteristic.

As expected, the low delay and jitter values of the Ethernet client enabled it to deliver a high QoE (Quality of Experience) [17] for both the video and the audio transmissions.

\section{B. Wifi 802.11}

Next we analyzed the performance of the WiFi client. Like in the Ethernet scenario, we built a table showing the minimum, maximum, mean, median and standard deviation of the OWD and IPDV experienced between the Wifi client and the flow-server, and evaluated using the video and audio streams in both the uplink and the downlink directions.

TABLE II

WIFI CLIENT METRICS STATISTICS

\begin{tabular}{|c|c|c|c|c|c|c|}
\hline \multicolumn{2}{|c|}{ Metric } & Max & Mean & Median & Min & Dev \\
\hline Video & OWD & 20.3739 & 4.6778 & 1.00303 & 0.5501 & 7.1284 \\
\cline { 2 - 7 } Up & IPDV & 1.4438 & $400 \mu \mathrm{s}$ & 0.06485 & -3.4165 & 2.1437 \\
\hline Audio & OWD & 3.3201 & 1.0819 & 0.58794 & 0.4001 & 1.7777 \\
\cline { 2 - 7 } Up & IPDV & 0.9828 & 0 & 0.01597 & -2.0391 & 1.4886 \\
\hline Video & OWD & 2.8499 & 1.2784 & 0.89598 & 0.5169 & 2.0727 \\
\cline { 2 - 7 } Down & IPDV & 0.6863 & 0 & 0.05913 & -1.7739 & 1.4879 \\
\hline Audio & OWD & 1.8242 & 0.8957 & 0.60296 & 0.5112 & 1.6164 \\
\cline { 2 - 7 } Down & IPDV & 0.6070 & 0 & 0.01812 & -1.5716 & 1.4064 \\
\hline
\end{tabular}
All values are in milliseconds, unless specified otherwise.

The MAC Layer of the WiFi link employs a positive acknowledgement system [1], i.e. retransmissions occur if a frame is not acknowledged within a given amount of time.
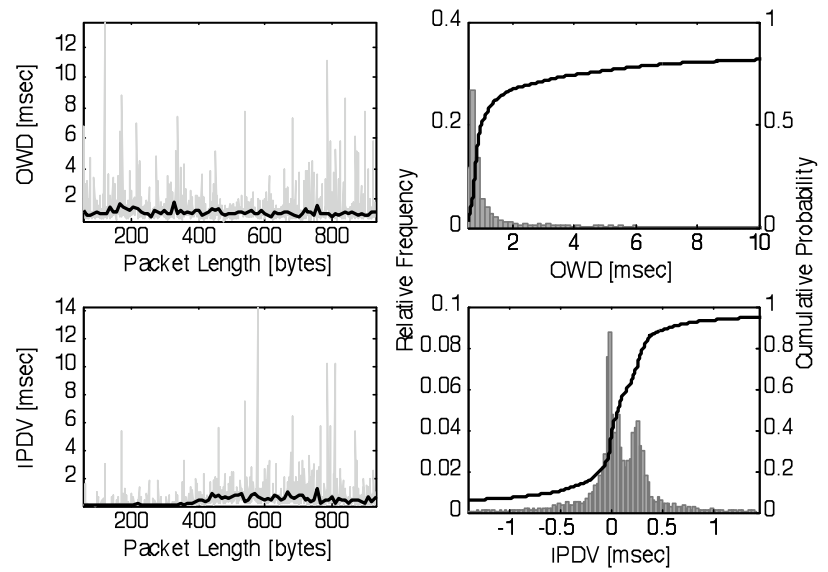

Fig. 2. OWD and IPDV for the Wifi downlink

This may explain the higher recorded maximum values for the one-way delay.

In the uplink direction the performance of the Wifi client was very good with respect to the Ethernet link, even outperforming the latter with a median value of $1.003 \mathrm{~ms}$ for the video stream and $0.587 \mathrm{~ms}$ for the audio stream, close to the minimum values for the computed metrics. The standard deviation values were higher, but in normal limits given the overall more erratic behavior of a radio link compared to a wired link. In terms of PLR, the link proved stable with $0 \%$ 
packet loss for the audio stream (again due to MAC Layer retransmissions) and as little as $0.034 \%$ for the video stream.

In the downlink direction the client experienced overall lower values for one-way delay. The packet loss ratio was $0.380 \%$ for the audio stream and $0.879 \%$ for the video stream.

Regarding the relation between the OWD, IPDV and packet size, the WiFi link demonstrated good stability on the downlink direction with nearly no variation at all. On the uplink direction the OWD characteristic remained unaffected by the packet length, and the IPDV showed increased values with packet lengths greater than 400 bytes (figure 2).

As expected from a client using the now mature 802.11 technology, the Quality of Experience was excellent on both the audio and video transmissions.

\section{WiMAX 802.16}

The performance of the WiMAX client was estimated using the same set of statistics for the OWD and the IPDV as shown in Table III.

TABLE III

WimAX CLIENT Metrics STATISTICS

\begin{tabular}{|c|c|c|c|c|c|c|}
\hline \multicolumn{2}{|c|}{ Metric } & Max & Mean & Median & Min & Dev \\
\hline \multirow{2}{*}{$\begin{array}{c}\text { Video } \\
\text { Up }\end{array}$} & OWD & 275.118 & 150.672 & 134.998 & 54.0566 & 77.686 \\
\hline & IPDV & 49.587 & 0 & 8.93307 & -80.051 & 35.0056 \\
\hline \multirow{2}{*}{$\begin{array}{c}\text { Audio } \\
\text { Up }\end{array}$} & $\mathrm{OV}$ & 4.9 & 07.5 & & 5 & 67.9576 \\
\hline & IPDV & 2 & $400 \mu \mathrm{s}$ & 4.6 & 78 & 27.819 \\
\hline \multirow{2}{*}{$\begin{array}{l}\text { Video } \\
\text { Down }\end{array}$} & OY & 158.7 & 78.5673 & & 55. & 34.5139 \\
\hline & IPD & 0.1601 & $-300 \mu \mathrm{s}$ & 006 & -22.082 & 9.5404 \\
\hline \multirow{2}{*}{$\begin{array}{l}\text { Audio } \\
\text { Down }\end{array}$} & OWD & 104.967 & 68.0588 & & & 23.1352 \\
\hline & IPDV & 8.6916 & 0 & 0.27585 & -16.799 & 8.5142 \\
\hline
\end{tabular}

All values are in milliseconds, unless specified otherwise.

The first thing we notice in the statistics is the discrepancy of the delay and jitter values between the downlink (from Base Station to Mobile Station) and uplink (from MS to BS) directions. The median, mean and minimum one-way delay values of the downlink are closely packed within two intervals spanning across 14 and $23 \mathrm{~ms}$ for the audio and video flow, respectively. In contrast, the same assumed intervals span across 65 and $96 \mathrm{~ms}$ for the uplink, indicating the presence of more outlier delay values. This is further consolidated by the higher standard deviation values of all distributions for the uplink metrics. We provide a visual description of this phenomenon in figures 3 and 4, where we present histograms and CDF plots of the one-way delay of packets from the video flows. Different performances in the uplink and downlink directions were also recorded in [10] where the authors have found much lower uplink bitrates compared to the corresponding downlink bitrates for distances above $2 \mathrm{~km}$. As they point out, the principal reason for this asymmetric behavior is that the power amplifier in the user terminal can only deliver a maximum of $20 \mathrm{dBm}$, compared to the $28 \mathrm{dBm}$ for the amplifier in the Base Station.

We next take a look at the different performances of this link when subjected to audio (CBR) and video (VBR) traffic. As expected, the higher overall throughput demand and the variable bitrate of the video flow have a negative effect on the link's performance. The recorded differences in the mean
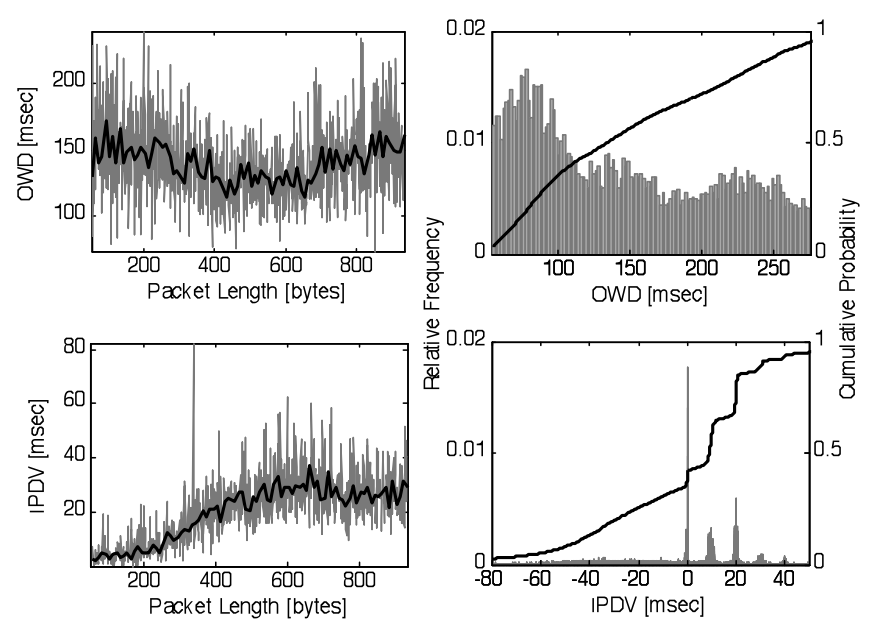

Fig. 3. OWD and IPDV for the WiMAX uplink
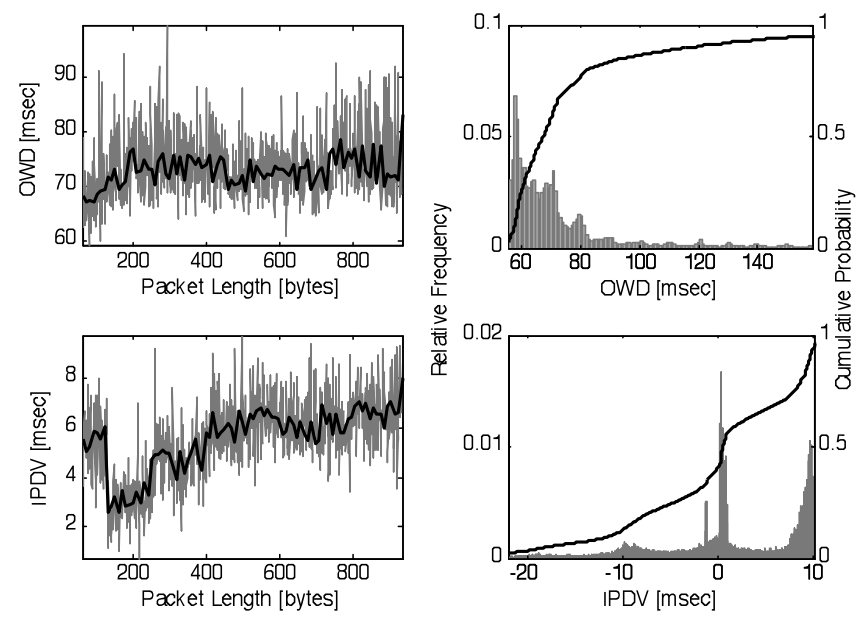

Fig. 4. OWD and IPDV for the WiMAX downlink

values of the one-way delay were $\sim 43 \mathrm{~ms}$ for the uplink and $\sim 10 \mathrm{~ms}$ for the downlink, consistent with the asymmetry described in the previous paragraph.

In order to better characterize this behavior we next analyzed the stability of the delay and the IPDV in respect to the packet size. In the uplink direction the OWD characteristic had a slight parabolic shape with an optimal packet size of 500 bytes, and the IPDV modulus showed a monotone increase from $\sim 5 \mathrm{~ms}$ at 100 bytes to $\sim 30 \mathrm{~ms}$ at 500 bytes and beyond (figure 3). An interesting result was recorded when analyzing the IPDV of the downlink: as shown in figure 4, we found high instability with small packet sizes ( $<125$ bytes).

Next we analyze the IPDV on the WiMAX link by computing its histograms. We can easily observe that the nature of both the uplink and the downlink delay variation is discrete (figures 3 and 4). The explanation for this discretization lies in our setup and in the Hybrid ARQ scheme employed by WiMAX's MAC layer for providing reliability. The link was operating in a TDD (Time Division Duplexing) mode, with a frame length of $5 \mathrm{~ms}$. This imposes a base extra delay of $10 \mathrm{~ms}$ for any frame that is retransmitted. Subsequent failures and retransmissions show up as delay variations multiple of the $10 \mathrm{~ms}$ base value (figure 3 ). This is consistent with the plots presented in [13], where the authors 
comparatively analyzed the original WiMAX HARQ scheme and their improved version. The average values of waiting time (the duration from the time the first copy of a data burst is received by the SS until the correct data burst is sent, in sequence, to the upper layer) found by the authors are always multiples of $10 \mathrm{~ms}$.

The multiple spikes present in the uplink IPDV histogram at 10, 20, 30 and even $40 \mathrm{~ms}$ show that for a significant number of frames, more than one retransmission was required.

In terms of Packet Loss Ratio the link demonstrated the same asymmetric behavior: $0.857 \%$ and $6.540 \%$ on the audio and video downlink compared to $3.237 \%$ and $10.621 \%$ on the audio and video uplink. This surprising result may be the effect of the experimental nature of our WiMAX implementation.

As for the QoE, this client offered satisfactory results for audio, but on the video channels, we experienced interruptions and image blockiness.

\section{UMTS}

The last step of our analysis was the UMTS (HSPA) client. Like in the WiMAX case, we observed a highly asymmetric tendency between the uplink and downlink computed metrics. Table IV shows the statistics for the video (Variable Bit Rate) stream.

TABLE IV

UMTS CLIENT METRICS STATISTICS

\begin{tabular}{|c|c|c|c|c|c|c|}
\hline \multicolumn{2}{|c|}{ Metric } & Max & Mean & Median & Min & Dev \\
\hline \multirow{2}{*}{$\begin{array}{c}\text { Video } \\
\text { Up }\end{array}$} & OWD & 258.251 & 153.877 & 95.3851 & 75.9034 & 263.481 \\
\hline & IPDV & 27.7925 & $-258 \mu \mathrm{s}$ & 1.0581 & -66.573 & 30.2289 \\
\hline \multirow{2}{*}{$\begin{array}{l}\text { Video } \\
\text { Down }\end{array}$} & OWD & 98.1764 & 74.2441 & 67.5008 & 50.0475 & 43.5242 \\
\hline & IPDV & 11.7511 & $-1.2 \mu \mathrm{s}$ & 1.8251 & -22.244 & 15.3494 \\
\hline
\end{tabular}

As the access setup most commonly offered by commercial UMTS providers consists of a dedicated channel with up to 64 $\mathrm{kbps}$ and $384 \mathrm{kbps}$ for uplink and downlink transmissions respectively, packets from the time interval when we used Isabel's higher quality profile were discarded and the UMTS client was setup not to send any audio data, thus avoiding congestion in the uplink direction.

On the uplink the UMTS link experienced a maximum (97.5 percentile) OWD of $258.25 \mathrm{~ms}$. As pointed out in [8], these outlier delay values are normal for a commercial UMTS link, if we take into account that under fully-loaded network conditions, the average latency for $3 \mathrm{G}$ data services can increase to beyond 1s. However the IPDV values on the UMTS uplink were smaller than those of the WiMAX uplink with a surprising $1.05 \mathrm{~ms}$ median value, and, the same interesting result regarding the discrete nature of the jitter histogram was observed (see figure 5). This is an effect of the UMTS link temporization employed at the RLC (Radio Link Control) sublayer of the MAC Layer. As the authors of [7] point out, the RLC layer always requires an integer number of TTI's (Transmission Time Intervals) to recover from a loss. When a lost frame is recovered RLC can deliver to upper layers several packets in one go. If these packets were transmitted at regular intervals, like in our case, discretization would occur. Another positive aspect was the stability of the
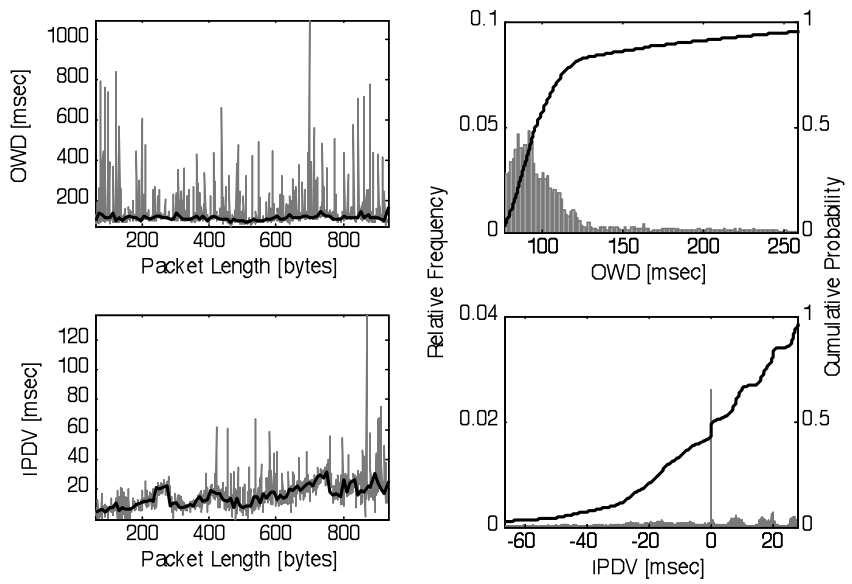

Fig. 5. OWD and IPDV for the UMTS uplink
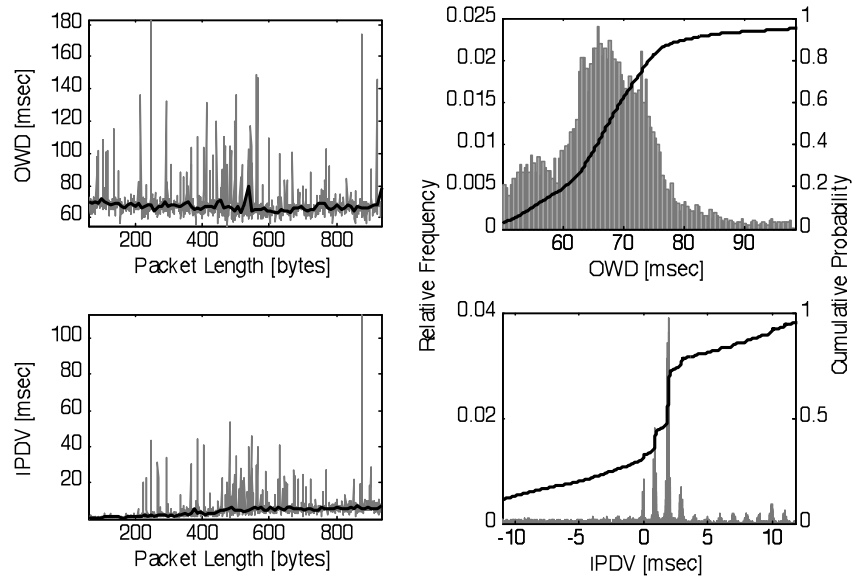

Fig. 6. OWD and IPDV for the UMTS downlink

uplink OWD and IPDV in respect to the packet size (see figure 5).

In the downlink direction the client performed better: the maximum OWD value was $98.17 \mathrm{~ms}$ (compared to $258 \mathrm{~ms}$ for the uplink), the mean value was close to the median, and the overall spread of the data was smaller $(43.524 \mathrm{~ms}$ standard deviation). The IPDV distribution had a mean value of $-1.2 \mu \mathrm{s}$, close to the ideal 0 and the harmonics were again observed in its histogram (figure 6). The link demonstrated good stability of the metrics in respect to the packet size: a flat characteristic for the OWD and a slight end-to-end increase in jitter.

Given the packet loss ratio, we can conclude that the ARQ mechanisms employed by the UMTS link are efficient. The recorded values were $0.250 \%$ and $0.242 \%$ for the downlink, respectively uplink. This is consistent with the results depicted in [7] were the authors have found that the packet losses never climbed over $0.5 \%$.

Although the bandwidth limitations of the UMTS link for data services imposed a special setup, the Quality of Experience was satisfactory with the lower quality settings.

\section{CONCLUSIONS}

In this paper we have presented a measurement-based comparison of the performance of three wireless access technologies: an IEEE 802.11 Wifi, an IEEE 802.16 WiMAX 
and a commercial HSPA-based UMTS link. Our analysis is based on statistical interpretations of principal delay measurements: one-way delay and inter-packet delay variation. Packet loss was also taken into account as we tried to emphasize on key asymmetries and differences between the access technologies.

Link reliability and stability in respect to packet size variations have proved that WLAN is a suitable technology for a generic videoconferencing application.

The WiMAX client lacked in reliability. High packet loss made this technology unsuitable for our Isabel application. An overall asymmetric downlink/uplink behavior was present, and we have seen uplink instability with varying packet sizes. We have also seen the jitter of the WiMAX link has a discrete nature because of frame retransmissions. The OWD and IPDV values were higher than those of UMTS and Wifi. The recorded PLR may be the effect of the experimental nature of our WiMAX implementation.

The UMTS link exhibited asymmetric behavior, a discrete nature of the delay variation was seen, but, under normal loading conditions, the commercial setup has shown very low jitter values. Extreme delay values can be the result of high network load. A probable better way to mitigate the low data bitrates offered by this link and the demands of high-quality videoconferencing would be the integration of these services with the inherent $3 \mathrm{G}$ video call capabilities.

\section{ACKNOWLEDGMENT}

The authors would like to thank Vodafone for providing part of the UMTS equipment and connectivity.

\section{REFERENCES}

[1] WLAN - IEEE Std 802.11-2007, "Wireless LAN Medium Access Control (MAC) and Physical Layer (PHY) Specifications"

[2] UMTS - "Radio interface protocol architecture," 3GPP, Technical Specification 25.301-v4.3.0 Release 4, June 2002.

[3] WiMAX - IEEE Std 802.16e-2005 and IEEE Std 802.16-2004/Cor12005, "Air Interface for Fixed and Mobile Broadband Wireless Access Systems"

[4] A Mishra, M Shin, W Arbaugh, "An empirical analysis of the IEEE 802.11 MAC layer handoff process" - ACM SIGCOMM Computer Communication Review, 2003

[5] Amit P. Jardosh et al. "Understanding link-layer behavior in highly congested IEEE 802.11b wireless networks", ACM SIGCOMM 2005

[6] Albert Cabellos Aparicio, René Serral-Gracià, Loránd Jakab, and Jordi Domingo-Pascual "Measurement Based Analysis of the Handover in a WLAN MIPv6 Scenario" PAM 2005, Boston, USA.

[7] Jose Manuel Cano-Garcia, Eva Gonzalez-Parada, and Eduardo Casilari, "Experimental Analysis and Characterization of Packet Delay in UMTS Networks", NEW2AN 2006

[8] Wee Lum Tan, Fung Lam, Wing Cheong Lau, "An Empirical Study on 3 G Network Capacity and Performance", Proceedings of IEEE Infocom May 2007

[9] Imperatore, P. Salvadori, E. Chlamtac, I. "Path Loss Measurements at 3.5 GHz: A Trial Test WiMAX Based in Rural Environment", TridentCom 2007

[10] Grondalen, O. Gronsund, P. Breivik, T. Engelstad, P, "Fixed WiMAX Field Trial Measurements and Analyses", Mobile and Wireless Communications Summit 2007

[11] Yousaf, Faqir Zarrar Daniel, Kai Wietfeld, Christian, "Performance Evaluation of IEEE 802.16 WiMAX Link With Respect to Higher Layer Protocols", ISCWS 2007
[12] Jurianto, Joe Hazra, S. K. Toh, S. H. Tan, W. M. Pathmasuntharam, Jaya $5.8 \mathrm{GHz}$ Fixed WiMAX Performance in a Sea Port Environment, IEEE VTC 2007

[13] M. Moh, T. Moh, Y. Shih, "On Enhancing WiMAX Hybrid ARQ: A Multiple-Copy Approach", Proceedings of IEEE CCNC 2008

[14] Isabel - http://www.agora-2000.com/pdfs/isabel_sheet_en.pdf

[15] LibPCAP - http://www.tcpdump.org/

[16] Internet2 Consortium: OWAMP - NTP Configuration http://e2epi.internet2.edu/owamp/details.html\#NTP (2004)

[17] Testing MPEG based IP video QoE/QoS http://www.shenick.com/pdfs/Testing_MPEG_IPTV_VOD_QOE.pdf 